\title{
A HAAR-RADO TYPE THEOREM FOR MINIMIZERS IN SOBOLEV SPACES
}

\section{Carlo Mariconda ${ }^{1}$ and Giulia Treu ${ }^{1}$}

Abstract. Let $u \in \phi+W_{0}^{1,1}(\Omega)$ be a minimum for

$$
I(v)=\int_{\Omega} g(x, v(x))+f(\nabla v(x)) \mathrm{d} x
$$

where $f$ is convex, $v \mapsto g(x, v)$ is convex for a.e. $x$. We prove that $u$ shares the same modulus of continuity of $\phi$ whenever $\Omega$ is sufficiently regular, the right derivative of $g$ satisfies a suitable monotonicity assumption and the following inequality holds

$$
\forall \gamma \in \partial \Omega \quad|u(x)-\phi(\gamma)| \leq \omega(|x-\gamma|) \quad \text { a.e. } x \in \Omega .
$$

This result generalizes the classical Haar-Rado theorem for Lipschitz functions.

Mathematics Subject Classification. 49K20.

Received July 23, 2009.

Published online October 28, 2010.

\section{INTRODUCTION}

The classical Haar-Rado Theorem [7] concerns the minimizers of the integral functional of the gradient $I(v)=\int_{\Omega} f(\nabla v(x)) \mathrm{d} x$ among the Lipschitz functions $u: \Omega \rightarrow \mathbb{R}$, where $\Omega$ is an open and bounded subset of $\mathbb{R}^{n}$. It asserts that if $f$ is strictly convex and $u$ is a minimizer of $I$ then, denoting by $\phi$ the restriction of $u$ to the boundary $\partial \Omega$ of $\Omega$, the Lipschitz rank of $u$ turns out to be equal to

$$
\sup \left\{\frac{|u(x)-\phi(\gamma)|}{|x-\gamma|}: x \in \Omega, \gamma \in \partial \Omega\right\} .
$$

In other terms, if one knows a priori that the minimizer $u$ is Lipschitz and that

$$
\forall x \in \Omega, \forall \gamma \in \partial \Omega \quad|u(x)-\phi(\gamma)| \leq K|x-\gamma|
$$

Keywords and phrases. Hölder, regularity, Lipschitz.

1 Dipartimento di Matematica Pura ed Applicata - Università Degli Studi di Padova, Via Trieste 63, 35121 Padova, Italy. maricond@math.unipd.it; treu@math.unipd.it 
then it turns out that

$$
\forall x, y \in \Omega \quad|u(y)-u(x)| \leq K|y-x|,
$$

i.e. that the Lipschitz constant of $u$ is bounded by $K$.

Among the applications of the Haar-Rado theorem, we quote the famous existence result of a minimizer among Lipschitz functions of the functional $I$ whenever the boundary datum satisfies a barrier condition, e.g. the Bounded Slope Condition of Hartman and Stampacchia [9].

The Bounded Slope Condition, that is in fact a quite restrictive assumption (see $[8,13]$ for detailed discussion), has been recently used and generalized in order to prove the regularity properties of minimizers that belong to Sobolev spaces $[3-5,10]$; their existence is guaranteed by Tonelli's theorem whenever the Lagrangian satisfies suitable growth assumptions. In $[3,4,10,12]$ the proofs are based on Comparison Principles, on a translation technique that goes back to [2] and on the extension of the boundary datum outside of the domain. The last step is straightforward when the boundary datum is Lipschitz, as in the case where $\phi$ satisfies the Bounded Slope Condition, but it is far to be trivial when we need to extend a function defined on $\partial \Omega$ to a function that is in a Sobolev space and has to share the same modulus of continuity with $\phi$. The extension of the boundary datum can be avoided if a Haar-Rado type theorem holds: this last remark together with the interest in obtaining regularity results under more general boundary conditions and for more general Lagrangians are the main motivations of the present paper.

The result that we present here is not only a reformulation of the classical Haar-Rado Theorem that encompasses the difficulty of working with Sobolev functions instead of Lipschitz ones, but it is a truly generalization of it. Indeed we take into account more general functionals of the form

$$
I(v)=\int_{\Omega} g(x, v(x))+f(\nabla v(x)) \mathrm{d} x,
$$

we do not assume the strict convexity of the functional that is needed in the classical Haar-Rado theorem and we deal with any modulus of continuity $\omega$ instead of just $\omega(t)=K|t|$, i.e. the Lipschitz one. This last matter allows us, for instance, to deal with the problem of the Hölder continuity of the minimizers, as in [12].

The lack of the strict convexity of $I$ has the counterpart that the minima may not be unique: assuming that $\Omega$ is sufficiently regular and that $\omega$ is a modulus of continuity of $\phi$ our result states that if $u$ is the minimum or the maximum of the minimizers of $I$ on $\phi+W^{1,1}(\Omega)$ and $g$ satisfies a monotonicity assumption $\left(\mathrm{H}_{\omega}\right)$ formulated below then $|u(y)-u(x)| \leq \omega(|y-x|)$ for a.e. $x, y \in \Omega$ once

$$
\forall \gamma \in \partial \Omega \quad|u(x)-\phi(\gamma)| \leq \omega(|x-\gamma|) \text { for a.e. } x \in \Omega
$$

The assumption $\left(\mathrm{H}_{\omega}\right)$ requires that $u \mapsto g(x, u)$ is convex, and that its right derivative $g_{v}^{+}(x, v)$ satisfies the monotonicity assumption

$$
v \geq u+\omega(|y-x|) \Longrightarrow g_{v}^{+}(y, v) \geq g_{v}^{+}(x, u) .
$$

It is worth mentioning that if $g(x, v)=g(v)$ does not depend on $x$, then the convexity of $g$ is enough for the validity of $\left(\mathrm{H}_{\omega}\right)$. For $\omega(t)=K|t|$ and $g$ of class $\mathcal{C}^{2}$ the assumption $\left(\mathrm{H}_{\omega}\right)$ is fulfilled if for instance

$$
\forall x \in \mathbb{R}^{n}, \forall v \in \mathbb{R} \quad\left|g_{x, v}(x, v)\right| \leq K g_{v, v}(x, v)
$$

for this particular modulus of continuity condition (1.2) appeared first in [14] for $g$ smooth, then in [10] in a nonsmooth setting; in these papers however it was used to obtain a regularity result for the minimizers of $I$ with no apparent relation with the Haar-Rado theorem. We point out that the functional related to the elasto-plastic torsion problem considered by Brezis and Sibony in [2] satisfies this assumption.

The proof of our result is based on several steps, most of which have an interest in themselves. In Section 2 we give an overview on how to work with the inequalities on the boundary of a domain in the trace sense. In Section 3 we show that if $u$ is the minimum or the maximum of the minimizers of $I$ that satisfies (1.1) then for all $h \in \mathbb{R}^{n}, u(x-h) \leq u(x)+\omega(|h|)$ on $\partial(\Omega \cap(h+\Omega))$ in the trace sense. Then assuming that $g(x, u)$ satisfies 
assumption $\left(\mathrm{H}_{\omega}\right)$ it turns out that $u(x-h)-\omega(|h|)$ is a subminimum of $I$ on $h+\Omega$. Finally, in Section 4 we prove the main result via a Comparison Principle for minimizers, a tool that we developed in the non strictly convex setting in [11], whose formulation and shorter proof is given here for the convenience of the reader.

As a final remark we mention that, under a mild geometric assumption on the epigraph of $f$, the main result holds for every minimizer of the functional $I$ instead of just the minimum/maximum of the minimizers.

A version of the results that we present here for solutions to a class of degenerate elliptic equations with some other applications will appear in a forthcoming paper.

\section{Notation AND BASIC DEFINITIONS}

In this paper $g: \mathbb{R}^{n} \times \mathbb{R} \rightarrow \mathbb{R}$ is a normal integrand, $f: \mathbb{R}^{n} \rightarrow \mathbb{R}$ is convex. $\Omega$ is a prescribed open and bounded subset of $\mathbb{R}^{n}$, whose closure is denoted by $\bar{\Omega}$.

The functional $I_{\Omega}$ (just $I$ when no ambiguity occurs) is

$$
I_{\Omega}(v)=\int_{\Omega} g(x, v(x))+f(\nabla v(x)) \mathrm{d} x
$$

As we fix a boundary datum $\phi$ we will always assume there is a function $v$ in $\phi+W_{0}^{1,1}(\Omega)$ such that $I_{\Omega}(v)$ is finite.

Definition 2.1. A function $u \in W^{1,1}(\Omega)$ is a sub-minimum (resp. super-minimum) of $I$ if $g(x, u)+f(\nabla u) \in$ $L^{1}(\Omega)$ and $I(v) \geq I(u)$ for every $v \in u+W_{0}^{1,1}(\Omega)$ with $v \leq u$ (resp. $v \geq u$ ) a.e. on $\Omega$. A function $u \in W^{1,1}(\Omega)$ is a minimizer of $I$ if it is both a sub-minimum and a super-minimum.

If $v$ and $w$ are functions then $v \wedge w$ (resp. $v \vee w$ ) stands for the pointwise minimum (resp. maximum) of $v$ and $w$. The restriction of a function $u$ to a subset $A$ of its domain is denoted by $u \mid A$. Finally, the scalar product in $\mathbb{R}^{n}$ is denoted by ".".

Definition 2.2 (modulus of continuity). A modulus of continuity is a positive continuous function $\omega:[0,+\infty[$ such that $\omega(0)=0$. A real valued function $\phi$ on a set $X$ is $\omega$-continuous if $|\phi(y)-\phi(x)| \leq \omega(|y-x|)$ for all $x, y \in X$.

\section{INEQUALITIES ON THE BOUNDARY}

\subsection{Inequalities in the trace sense}

In this section $D$ is an open and bounded subset of $\mathbb{R}^{n}$. The following notion is crucial for our comparison principles.

Definition 3.1 (inequalities in the trace sense). Let $u, v \in W^{1,1}(D)$. We say that $u \leq v$ in $\partial D$ in the trace sense if $u \wedge v \in u+W_{0}^{1,1}(D)$ or, equivalently, if $u \vee v$ is in $v+W_{0}^{1,1}(D)$.

We give now some useful criteria that ensure the validity of an inequality in the trace sense.

Proposition 3.1. Let $u, v \in W^{1,1}(D)$. Then $u \leq v$ in $\partial D$ in the trace sense if one of the following assumption hold:

(a) $u, v \in \mathcal{C}(\bar{D})$ and $u(\gamma) \leq v(\gamma)$ for every $\gamma \in \partial D$;

(b) $u \leq v$ a.e. in $D$;

(c) there are two sequences $u_{k}, v_{k} \in \mathcal{C}(\bar{D}) \cap W^{1,1}(D)$ such that $u_{k}$ converges to $u$ and $v_{k}$ converges to $v$ in $W^{1,1}(D)$ and moreover $u_{k}(\gamma) \leq v_{k}(\gamma)$ for every $\gamma \in \partial D$

(d) the boundary of $D$ is regular, $D$ is a relatively compact subset of an open subset $A$ of $\mathbb{R}^{n}$, u,v are the restrictions of $\bar{u}, \bar{v} \in W_{\mathrm{loc}}^{1,1}(A)$ and $\bar{u} \leq \bar{v}$ a.e. on $A \backslash D$. 
Proof. (a) If $u(\gamma) \leq v(\gamma)$ on $\partial D$ then $u \wedge v-u=0$ pointwise on $\partial D$ : follows from [1], Theorem IX.17, together with [1], Remark 20, Chapter IX, that $u \wedge v-u \in W_{0}^{1,1}(D)$.

(b) If $u \leq v$ a.e. in $D$ then $u \wedge v-u=0$ a.e. in $D$.

Assume (c); it follows from (a) that $u_{k} \wedge v_{k}-u_{k} \in W_{0}^{1,1}(D)$. Since $u_{k} \wedge v_{k}-u_{k}$ converges to $u \wedge v-u$ the claim follows from the closure of $W_{0}^{1,1}(D)$.

(d) Since $\bar{u} \wedge \bar{v}-\bar{u} \in W_{\text {loc }}^{1,1}(A)$ and $\bar{u} \wedge \bar{v}-\bar{u}=0$ a.e. in $A \backslash D$ then $\bar{u} \wedge \bar{v}-\bar{u} \in W_{0}^{1,1}(A)$. It follows from [1], Proposition IX.18, that the extension $w$ of $\bar{u} \wedge \bar{v}-\bar{u}$ defined by

$$
w(x)= \begin{cases}(\bar{u} \wedge \bar{v}-\bar{u})(x) & \text { if } x \in A \\ 0 & \text { if } x \notin A\end{cases}
$$

belongs to $W^{1,1}\left(\mathbb{R}^{n}\right)$. Now $w$ is also the extension of $u \wedge v-u$ equal to 0 out of $D$ and $\partial D$ is regular. Again by [1], Proposition IX.18, this implies that $u \wedge v-u$ is in $W_{0}^{1,1}(D)$.

\subsection{Inequalities between trace functions in Lipschitz domains}

There is another notion of inequalities on the boundary, involving the trace functions, that is useful when the domain is regular. We show that in this case the two notions of inequality in the trace sense formulated above and of the trace inequalities given here coincide.

We assume here that $\partial \Omega$ is Lipschitz. Let us recall the result on the existence of a trace function, we refer to [6] for the details. We denote by $\mathrm{L}_{\mathcal{H}^{n-1}}^{1}(\partial \Omega)$ the set of Lebesgue integrable functions on $\partial \Omega$ with respect to the $(n-1)$-dimensional Hausdorff measure $\mathcal{H}^{n-1}$.

Theorem 3.1. There exists a linear, bounded operator $\operatorname{Tr}^{\Omega}: W^{1,1}(\Omega) \longrightarrow \mathrm{L}_{\mathcal{H}^{n-1}}^{1}(\partial \Omega)$ named the trace operator such that $\operatorname{Tr}^{\Omega} u(\gamma)=u(\gamma)$ for all $\gamma \in \partial \Omega$ whenever $u \in(\bar{\Omega}) \cap W^{1,1}(\Omega)$. Moreover

$$
W_{0}^{1,1}(\Omega)=\left\{u \in W^{1,1}(\Omega): \operatorname{Tr}^{\Omega} u=0\right\} .
$$

The following characterization of the functions that are traces will be used in the sequel.

Proposition 3.2 (characterization of the trace functions). Let $\phi \in \mathrm{L}_{\mathcal{H}^{n-1}}^{1}(\partial \Omega)$. The following claims are equivalent:

(i) $\phi=\operatorname{Tr}^{\Omega} u$ for some $u \in W^{1,1}(\Omega)$;

(ii) there is a sequence $\left(u_{n}\right)_{n}$ of Lipschitz functions on $\bar{\Omega}$ such that $u_{n} \rightarrow u$ in $W^{1,1}(\Omega)$ and $u_{n \mid \partial \Omega} \rightarrow \phi$ in $\mathrm{L}_{\mathcal{H}^{n-1}}^{1}(\partial \Omega)$;

(iii) for $\mathcal{H}^{n-1}$-a.e. $\gamma \in \partial \Omega$ we have

$$
\lim _{r \rightarrow 0^{+}} f_{B_{r}(\gamma) \cap \Omega}|\phi(\gamma)-u(x)| \mathrm{d} x=0 .
$$

Proof. (i) $\Rightarrow$ (ii): Assume that $\phi=\operatorname{Tr}^{\Omega} u$ : let $\left(u_{n}\right)_{n}$ in $\mathcal{C}^{1}(\bar{\Omega})$ such that $u_{n} \rightarrow u$ in $W^{1,1}(\Omega)$; the continuity of the trace operator yields

$$
\phi=\operatorname{Tr}^{\Omega} u=\lim _{n} \operatorname{Tr}^{\Omega} u_{n}=\lim _{n} u_{n \mid \partial \Omega} \text { in } \operatorname{L}_{\mathcal{H}^{n-1}}^{1}(\partial \Omega) .
$$

Conversely, assume that there are $u_{n} \in \operatorname{Lip}(\bar{\Omega})$ with $u_{n} \rightarrow u$ in $W^{1,1}(\Omega)$ and $u_{n \mid \partial \Omega} \rightarrow \phi$ in $\mathrm{L}_{\mathcal{H}^{n-1}}^{1}(\partial \Omega)$. Again, the continuity of the trace operator implies that $\operatorname{Tr}^{\Omega} u_{n}$ converges to $\operatorname{Tr}^{\Omega} u$ in $\mathrm{L}_{\mathcal{H}^{n-1}}^{1}(\partial \Omega)$; since $\operatorname{Tr}^{\Omega} u_{n}=u_{n \mid \partial \Omega}$ the two limits coincide, and it follows that $\operatorname{Tr}^{\Omega} u=\phi$. The proof of the equivalence (i) $\Rightarrow$ (iii) can be found in $[6]$. 
Remark 3.1. It follows from (iii) of Proposition 3.2 that if $u \in W^{1,1}(\Omega)$ then

$$
\operatorname{Tr}^{\Omega} u(\gamma)=\lim _{r \rightarrow 0^{+}} f_{B_{r}(\gamma) \cap \Omega} u(x) \mathrm{d} x \text { a.e. }
$$

The monotonicity of the trace follows then directly.

Corollary 3.1. Monotonicity of the trace Let $u, v \in W^{1,1}(\Omega)$ be such that $u \leq v$ a.e. in $\Omega$. Then $\operatorname{Tr}^{\Omega} u \leq \operatorname{Tr}^{\Omega} v$ $\mathcal{H}^{n-1}$-a.e.

In the case where $\partial \Omega$ is Lipschitz the notion of inequality of functions in the trace sense as in Definition 3.1 is hopefully equivalent to the a.e. inequality among traces.

Proposition 3.3. Let $\partial \Omega$ be Lipschitz and $u, v \in W^{1,1}(\Omega)$. Then $u \leq v$ in the trace sense, i.e. $u \vee v \in$ $v+W_{0}^{1,1}(\Omega)$, if and only if $\operatorname{Tr}^{\Omega}(u) \leq \operatorname{Tr}^{\Omega}(v) \mathcal{H}^{n-1}$-a.e. on $\partial \Omega$.

Proof. It is clearly enough to prove the claim when $v=0$. Assume that $u \leq 0$ in the trace sense, namely that $u \vee 0 \in W_{0}^{1,1}(\Omega)$. Then by (3.1) $\operatorname{Tr}^{\Omega}(u \vee 0)=0$, the monotonicity of the trace operator then implies that $\operatorname{Tr}^{\Omega} u \leq \operatorname{Tr}^{\Omega}(u \vee 0)=0$ a.e. Conversely assume that $\operatorname{Tr}^{\Omega} u \leq 0 \mathcal{H}^{n-1}$-a.e. on $\partial \Omega$. Let $\left(u_{n}\right)_{n}$ be a sequence in $\operatorname{Lip}(\bar{\Omega})$ such that $u_{n} \rightarrow u$ in $W^{1,1}(\Omega)$ and $u_{n \mid \partial \Omega} \rightarrow \operatorname{Tr}^{\Omega} u$ in $\mathrm{L}_{\mathcal{H}^{n-1}}^{1}(\partial \Omega)$. By taking a subsequence we may assume that $u_{n \mid \partial \Omega} \rightarrow \operatorname{Tr}^{\Omega} \mathcal{H}^{n-1}$-a.e. on $\partial \Omega$. Now $u_{n} \vee 0 \rightarrow u \vee 0$ in $W^{1,1}(\Omega)$ whereas $u_{n} \vee 0 \in \operatorname{Lip}(\bar{\Omega})$ and $\left(u_{n} \vee 0\right)_{\mid \partial \Omega} \rightarrow \operatorname{Tr}^{\Omega} u \vee 0=0$ a.e. on $\partial \Omega$; it follows from Proposition 3.3 that $\operatorname{Tr}^{\Omega}(u \vee 0)=0$ so that, again by (3.1), $u \vee 0 \in W_{0}^{1,1}(\Omega)$ meaning that $u \leq 0$ in the trace sense.

Remark 3.2. The claim of Proposition 3.3 would also follow from the fact that, for $u, v \in W^{1,1}(\Omega)$,

$$
\operatorname{Tr}^{\Omega}(u \wedge v)=\operatorname{Tr}^{\Omega} u \wedge \operatorname{Tr}^{\Omega} v, \quad \operatorname{Tr}^{\Omega}(u \vee v)=\operatorname{Tr}^{\Omega} u \vee \operatorname{Tr}^{\Omega} v
$$

We end this section with a result on the trace on the intersection of two domains that will be used later.

Lemma 3.1. Let $A, B$ be two open bounded subsets of $\mathbb{R}^{n}$ such that $\partial A, \partial B, \partial(A \cap B)$ are Lipschitz. Let $u \in W^{1,1}(A)$. Then $\operatorname{Tr}^{A} u=\operatorname{Tr}^{A \cap B} u$ a.e. on $\partial A \cap \partial(A \cap B)$.

Proof. Let $\left(u_{n}\right)_{n}$ in $\operatorname{Lip}(\bar{\Omega})$ such that $u_{n} \rightarrow u$ in $W^{1,1}(A)$ with $u_{n \mid \partial A} \rightarrow \operatorname{Tr}^{A} u$ in $\operatorname{L}_{\mathcal{H}^{n-1}}^{1}(\partial A)$, by taking a subsequence we may assume that the convergence holds a.e. in $\partial A$. Now $u_{n} \rightarrow u$ in $W^{1,1}(A \cap B)$ : the continuity of the trace operator then implies that $u_{n \mid \partial(A \cap B)}=\operatorname{Tr}^{A \cap B} u_{n} \rightarrow \operatorname{Tr}^{A \cap B} u$ in $\mathrm{L}_{\mathcal{H}^{n-1}}^{1}(\partial(A \cap B))$; again we may assume that the convergence holds a.e. The uniqueness of the (pointwise) limit yields the claim.

Remark 3.3. In Lemma 3.1 it is not enough to assume the Lipschitz regularity of $\partial A$ and $\partial B$. Indeed it is easy to build some examples in which these two sets are Lipschitz but $\partial(A \cap B)$ is not.

\section{Minimality Properties of translates}

We consider the following assumption. We denote by $g_{v}^{+}(x, v)$ the right derivative of $g$ with respect to the second variable.

Assumption $\left(\mathrm{H}_{h, c}\right)$. Let $h \in \mathbb{R}^{n}$ and $c \in \mathbb{R}$. The function $g: \mathbb{R}^{n} \times \mathbb{R} \rightarrow \mathbb{R}$ is convex in the second variable. We assume that

$$
\forall x \in \mathbb{R}^{n}, \forall u, v \in \mathbb{R} \quad v \geq u+c \Rightarrow g_{v}^{+}(x \pm h, v) \geq g_{v}^{+}(x, u) .
$$

Let $\omega$ be a modulus of continuity on $\mathbb{R}^{n}$. In the next section we will be concerned with Assumption $\left(\mathrm{H}_{h, c}\right)$ when $c=\omega(|h|)$ for all $h \in \mathbb{R}^{n}$; it is fulfilled if the following Assumption $\left(\mathrm{H}_{\omega}\right)$ holds. 
Assumption $\left(\mathrm{H}_{\omega}\right)$. The function $g: \mathbb{R}^{n} \times \mathbb{R} \rightarrow \mathbb{R}$ is measurable and convex in the second variable. Denoting by $g_{v}^{+}$the right derivative of $g$ with respect to the second variable, we assume that

$$
\forall x, y \in \mathbb{R}^{n}, \forall u, v \in \mathbb{R} \quad v \geq u+\omega(|y-x|) \Rightarrow g_{v}^{+}(y, v) \geq g_{v}^{+}(x, u)
$$

Remark 4.1. It is worth noticing that Assumption $\left(\mathrm{H}_{\omega}\right)$ is fulfilled when the function $g(x, v)=g(v)$ is convex and does not depend on $x$.

We state a condition under which Assumption $\left(\mathrm{H}_{\omega}\right)$ is fulfilled in the case where $\omega$ is a Lipschitz modulus of continuity, i.e. $\omega(t) \leq K|t|$.

Proposition 4.1. Let $K>0$; assume that $g$ is of class $\mathcal{C}^{2}$ and such that

$$
\forall x \in \mathbb{R}^{n}, \forall v \in \mathbb{R}, \quad\left|g_{x, v}(x, v)\right| \leq K g_{v, v}(x, v) .
$$

Then $g$ satisfies Assumption $\left(\mathrm{H}_{\omega}\right)$ where $\omega(t)=K|t|$.

Proof. Assume that $v \geq u+K|y-x|$. Now there are $(\xi, \zeta)$ such that

$$
g_{v}(y, v)-g_{v}(x, u)=g_{x, v}(\xi, \zeta) \cdot(y-x)+g_{v, v}(\xi, \zeta)(v-u)
$$

Our condition implies that $g_{v, v} \geq 0$, thus

$$
\begin{aligned}
g_{v}(y, v)-g_{v}(x, u) & \geq g_{x, v}(\xi, \zeta) \cdot(y-x)+g_{v, v}(\xi, \zeta) K|x-y| \\
& \geq-\left|g_{x, v}(\xi, \zeta)\right||y-x|+g_{v, v}(\xi, \zeta) K|x-y| \\
& =|x-y|\left(K g_{v, v}(\xi, \zeta)-\left|g_{x, v}(\xi, \zeta)\right|\right) \geq 0
\end{aligned}
$$

proving the claim.

Remark 4.2. In the case where $\omega(t)=K|t|$ some conditions similar to those stated above where formulated in [14] and, in a nonsmooth setting, in [10]. In both papers, however, they were used to establish Lipschitz regularity results for the minimizers of $I$ with no apparent connections to the result that we formulate in the present paper.

Theorem 4.1. Let $h \in \mathbb{R}^{n}$ and $c \in \mathbb{R}$ and assume that $g$ satisfies Assumption $\left(\mathrm{H}_{h, c}\right)$. Let $u$ be a sub-minimum of $I$ then $u(y-h)-c$ is a sub-minimum of $I_{h+\Omega}$. Analogously, if $u$ is a super-minimum of $I$ then $u(y+h)+c$ is a super-minimum of $I_{-h+\Omega}$.

Proof. Assume that $u$ is a super-minimum of $I$. Let $v \in W^{1,1}(\Omega)$ be such that

$$
v(y) \geq u(y+h)+c \text { a.e. on }-h+\Omega, \quad v(y)=u(y+h)+c \text { on } \partial(-h+\Omega)
$$

Then $v(x-h)-c \geq u(x)$ a.e. on $\Omega$ and $v(x-h)-c=u(x)$ on $\partial \Omega$. Since $u$ is a super-minimum then $I(v(x-h)-c) \geq I(u)$; we wish to prove that the inequality $I_{-h+\Omega}(v) \geq I_{-h+\Omega}(u(y+h)+c)$ holds true. Now

$$
\begin{aligned}
I_{-h+\Omega}(v) & =\int_{-h+\Omega} g(y, v(y))+f(\nabla v(y)) \mathrm{d} y \\
& =\int_{\Omega} g(x-h, v(x-h))+f(\nabla v(x-h)) \mathrm{d} x \\
& =\int_{\Omega} g(x-h, v(x-h)) \mathrm{d} x-\int_{\Omega} g(x, v(x-h)-c) \mathrm{d} x+I_{\Omega}(v(x-h)-c) .
\end{aligned}
$$


Analogously

$$
\begin{aligned}
I_{-h+\Omega}(u(y+h)+c) & =\int_{-h+\Omega} g(y, u(y+h)+c)+f(\nabla u(y+h)) \mathrm{d} y \\
& =\int_{\Omega} g(x-h, u(x)+c) \mathrm{d} x-\int_{\Omega} g(x, u(x)) \mathrm{d} x+I_{\Omega}(u) .
\end{aligned}
$$

Since $u$ is a super-minimum then $I_{\Omega}(v(x-h)-c) \geq I_{\Omega}(u)$ : therefore it is enough to show that

$$
g(x-h, v(x-h))-g(x, v(x-h)-c) \geq g(x-h, u(x)+c)-g(x, u(x)) \text { a.e. on } \Omega
$$

or, equivalently,

$$
g(x-h, v(x-h))-g(x-h, u(x)+c) \geq g(x, v(x-h)-c)-g(x, u(x)) \text { a.e. on } \Omega .
$$

For this purpose fix $x \in \Omega$ such that all the above quantities are defined and finite. Set, for $t \in[u(x)+c, v(x-h)]$, $\psi(t)=g(x-h, t), \phi(t)=g(x, t-c)$. Then $\psi, \phi$ have right derivatives $\psi_{+}^{\prime}(t)=g_{v}^{+}(x-h, t), \phi_{+}^{\prime}(t)=g_{v}^{+}(x, t-c)$; since the difference $t-(t-c)=c \geq c$ the assumption $\left(\mathrm{H}_{h, c}\right)$ implies that $g_{v}^{+}(x-h, t) \geq g_{v}^{+}(x, t-c)$ and thus $\psi_{+}^{\prime}(t) \geq \phi_{+}^{\prime}(t)$ on $[u(x)+c, v(x-h)]$. The mean value theorem thus yields

$$
\psi(v(x-h))-\psi(u(x)+c) \geq \phi(v(x-h))-\phi(u(x)+c)
$$

which is exactly (4.1), proving the claim.

\section{A Rado-HaAr type theorem}

The next result was proven, just for minima and not as here for sub/super-minima, in [11], Theorem 2.1. We give here the proof both for the convenience of the reader and because we use here an elegant and concise argument that was suggested to the authors by Sergio Solimini in a personal communication. It is based on the so called Stampacchia Principle that we do not found however in the literature. We formulate it here for the convenience of the reader, its proof being straightforward.

Lemma 5.1. Let $L: \Omega \times \mathbb{R} \times \mathbb{R}^{n} \rightarrow \mathbb{R}$ be a normal integrand and set

$$
\mathcal{L}(u)=\int_{\Omega} L(x, u, \nabla u) \mathrm{d} x
$$

Assume that $u, v \in W^{1,1}(\Omega)$ are such that $L(x, u, \nabla u), L(x, v, \nabla v) \in L^{1}(\Omega)$. Then

$$
\mathcal{L}(u)+\mathcal{L}(v)=\mathcal{L}(u \wedge v)+\mathcal{L}(u \vee v)
$$

Let us recall that if the functional $I$ is not strictly convex the minima may not be unique. As we showed in [11], given a trace function $\phi$ on $\partial \Omega$, the minimum and the maximum of the minimizers of $I_{\Omega, \phi}$ exist if $f$ is superlinear.

Theorem 5.1. Assume that $u$ is the minimum of the minimizers of $I_{\Omega, \phi}$, where $\phi$ is a given trace function on $\partial \Omega$. Let $v$ be a super-minimum of $I$ with $u \leq v$ on $\partial \Omega$. Then $u \leq v$ a.e. on $\Omega$; in other words $u$ satisfies the Comparison Principle from below. Analogously, the maximum of the minimizers satisfies the Comparison Principle from above.

Proof. By Lemma 5.1 we have

$$
I(u)+I(v)=I(u \wedge v)+I(u \vee v)
$$

Since $u \leq v$ on $\partial \Omega$ then

$$
u \wedge v=u \text { on } \partial \Omega \quad u \vee v=v \text { on } \partial \Omega .
$$


Since $u$ is a minimizer and $v$ is a super-minimum then

$$
I(u \wedge v) \geq I(u) \quad I(u \vee v) \geq I(v)
$$

so that, by (5.1), the latter inequalities turn out to be equalities. Therefore $u \wedge v$ is a minimizer of $I$ with $u \wedge v=u$ on $\partial \Omega$ : the minimality of $u$ implies that $u \leq u \wedge v$ a.e. so that $u \leq u \wedge v \leq v$ a.e. on $\Omega$. The second part of the claim follows similarly.

The next result is a version of the Haar-Rado Theorem [7], in the setting of Sobolev functions.

Theorem 5.2 (Haar-Rado type). Let $\omega:[0,+\infty[\rightarrow[0,+\infty[$ be a modulus of continuity. Let $\phi$ be a function in $W^{1,1}(\Omega)$ that is $\omega$-continuous on $\bar{\Omega}$ and let $u \in \phi+W_{0}^{1,1}(\Omega)$ be the minimum or the maximum of the minimizers of $I$. Assume that one of the following conditions holds:

$\left(\mathrm{H}_{1}\right) u, \phi \in \mathcal{C}(\bar{\Omega})$ and

$$
\forall \gamma \in \partial \Omega, \quad \forall x \in \Omega \quad|u(x)-\phi(\gamma)| \leq \omega(|x-\gamma|) ;
$$

$\left(\mathrm{H}_{2}\right) \Omega \cap(h+\Omega)$ is regular for all $h \in \mathbb{R}^{n}$; moreover

$$
\forall \gamma \in \partial \Omega \quad|u(x)-\phi(\gamma)| \leq \omega(|x-\gamma|) \quad \text { a.e. } x ;
$$

$\left(\mathrm{H}_{3}\right) \Omega \cap(h+\Omega)$ is regular for all $h \in \mathbb{R}^{n}$; moreover there exist $\ell_{1}, \ell_{2} \in \phi+W_{0}^{1,1}(\Omega)$ that are $\omega$-continuous on $\bar{\Omega}$ and such that

$$
\ell_{1}(x) \leq u(x) \leq \ell_{2}(x) \text { a.e. on } \Omega \text {. }
$$

Then $|u(y)-u(x)| \leq \omega(|y-x|)$ for every Lebesgue points $x$ and $y$ of $u$.

For $h \in \mathbb{R}^{n}$ and $u$ a function on $\Omega$ we denote by $u_{h}$ the function defined on $-h+\Omega$ by $u_{h}(x)=u(x+h)$ and we set $\Omega_{h}=(-h+\Omega) \cap \Omega$.

Lemma 5.2. Let $\phi$ be a function in $W^{1,1}(\Omega)$ that is $\omega$-continuous on $\bar{\Omega}$ and let $u \in \phi+W_{0}^{1,1}(\Omega)$. Assume that one the conditions $\left(\mathrm{H}_{1}\right),\left(\mathrm{H}_{2}\right)$ or $\left(\mathrm{H}_{3}\right)$ stated in Theorem 5.2 holds. Then

$$
u_{h} \leq u+\omega(|h|) \text { on } \partial \Omega_{h}
$$

in the trace sense.

Proof. Assume that $\left(\mathrm{H}_{1}\right)$ holds. Let $\gamma \in \partial \Omega_{h}$; it is not restrictive to assume that $\gamma \in \partial \Omega$. Either $\gamma \in-h+\Omega$ and $u_{h}(\gamma)-u(\gamma)=u(\gamma-h)-\phi(\gamma) \leq \omega(|\gamma-h-\gamma|)=\omega(|h|)$ or $\gamma \in \partial(-h+\Omega)$ and $u_{h}(\gamma)-u(\gamma)=\phi(\gamma-h)-\phi(\gamma) \leq$ $\omega(|h|)$ by the $\omega$-continuity of $\phi$ : in both cases $u_{h}(\gamma)-u(\gamma) \leq \omega(|h|)$ for every $\gamma \in \partial \Omega_{h}$. Proposition 3.1(a) yields the claim.

Assume that $\left(\mathrm{H}_{2}\right)$ holds; we show that, for $\mathcal{H}^{n-1}$-almost every $\gamma \in \partial\left(\Omega \cap \Omega_{h}\right)$,

$$
\lim _{r \rightarrow 0} f_{B_{r}(\gamma) \cap \Omega \cap \Omega_{h}} u_{h}(x)-u(x) \mathrm{d} x \leq \omega(|h|) .
$$

Let $\gamma \in \partial\left(\Omega \cap \Omega_{h}\right)$; it is not restrictive to assume that $\gamma \in \partial \Omega$. 
Two cases may occur:

(a) $\gamma \in \Omega_{h}$ so that $B_{r}(\gamma) \cap \Omega \cap \Omega_{h}=B_{r}(\gamma) \cap \Omega$ for $r$ sufficiently small. Since for a.e. $x \in \Omega \cap \Omega_{h}$ we have $u_{h}(x)-u(x)=u_{h}(x)-\phi(\gamma)+\phi(\gamma)-u(x)$ then

$$
\begin{aligned}
f_{B_{r}(\gamma) \cap \Omega \cap \Omega_{h}} u_{h}(x)-u(x) \mathrm{d} x & \leq f_{B_{r}(\gamma) \cap \Omega \cap \Omega_{h}} u_{h}(x)-\phi(\gamma) \mathrm{d} x+f_{B_{r}(\gamma) \cap \Omega \cap \Omega_{h}} \phi(\gamma)-u(x) \mathrm{d} x \\
& =f_{B_{r}(\gamma) \cap \Omega \cap \Omega_{h}} u_{h}(x)-\phi(\gamma) \mathrm{d} x+f_{B_{r}(\gamma) \cap \Omega} \phi(\gamma)-u(x) \mathrm{d} x .
\end{aligned}
$$

Now by $\left(\mathrm{H}_{2}\right) u_{h}(x)-\phi(\gamma)=u(x-h)-\phi(\gamma) \leq \omega(|x-h-\gamma|)$ a.e. and thus

$$
f_{B_{r}(\gamma) \cap \Omega \cap \Omega_{h}} u_{h}(x)-u(x) \mathrm{d} x \leq f_{B_{r}(\gamma) \cap \Omega \cap \Omega_{h}} \omega(|x-h-\gamma|) \mathrm{d} x+f_{B_{r}(\gamma) \cap \Omega} \phi(\gamma)-u(x) \mathrm{d} x
$$

and $f_{B_{r}(\gamma) \cap \Omega \cap \Omega_{h}} \omega(|x-h-\gamma|) \mathrm{d} x \rightarrow \omega(|\gamma-h-\gamma|)=\omega(|h|)$ as $r \rightarrow 0$ whereas $f_{B_{r}(\gamma) \cap \Omega} \phi(\gamma)-u(x) \mathrm{d} x \rightarrow 0$ as $r \rightarrow 0$ unless $\gamma$ belongs to a $\mathcal{H}^{n-1}$-negligible subset of $\partial \Omega$, proving (5.6).

(b) $\gamma \in \partial \Omega_{h}$ so that $\gamma-h \in \partial \Omega$ and, as above, $\gamma \in \partial \Omega$. Again by dropping out a $\mathcal{H}^{n-1}$-negligible subset of $\partial \Omega$, we may assume from Lemma 3.1 that

$$
\operatorname{Tr}^{\Omega} u(\gamma)=\operatorname{Tr}^{\Omega \cap \Omega_{h}} u(\gamma)=\phi(\gamma), \quad \operatorname{Tr}^{\Omega_{h}} u(\gamma-h)=\operatorname{Tr}^{\Omega \cap \Omega_{h}} u(\gamma-h)=\phi(\gamma-h)
$$

Now for a.e. $x \in \Omega \cap \Omega_{h}$ we have

$$
u_{h}(x)-u(x)=u_{h}(x)-\phi(\gamma-h)+\phi(\gamma-h)-\phi(\gamma)+\phi(\gamma)-u(x)
$$

and

$$
f_{B_{r}(\gamma) \cap \Omega \cap \Omega_{h}} u_{h}(x)-\phi(\gamma-h) \mathrm{d} x \rightarrow 0 \quad f_{B_{r}(\gamma) \cap \Omega \cap \Omega_{h}} \phi(\gamma)-u(x) \mathrm{d} x \rightarrow 0
$$

as $r \rightarrow 0$; moreover $\phi(\gamma-h)-\phi(\gamma) \leq \omega(|h|)$ since $\phi$ is $\omega$-continuous. Therefore

$$
\lim _{r \rightarrow 0} f_{B_{r}(\gamma) \cap \Omega \cap \Omega_{h}} u_{h}(x)-u(x) \mathrm{d} x \leq \omega(|h|)
$$

a.e. on $\partial\left(\Omega \cap \Omega_{h}\right)$ proving (5.6).

The conclusion of Theorem 5.2 under $\left(\mathrm{H}_{3}\right)$ follows directly from the previous case since, if (5.4) holds then for every $\gamma \in \partial \Omega$ and for a.e $x$ in $\Omega$ we have

$$
u(x)-\phi(\gamma) \leq \ell_{2}(x)-\phi(\gamma)=\ell_{2}(x)-\ell_{2}(\gamma) \leq \omega(|x-\gamma|)
$$

and, analogously,

ensuring the validity of (5.3).

$$
-\omega(|x-\gamma|) \leq \ell_{1}(\gamma)-\ell_{1}(x) \leq \phi(\gamma)-u(x)
$$

Proof of Theorem 5.2. Assume that $u$ is the maximum of the minimizers of $I$. Fix $h$ in $\mathbb{R}^{n}$ : we know from Lemma 5.2 that $u_{h}-\omega(|h|) \leq u$ on $\partial\left(\Omega \cap \Omega_{h}\right)$. By Theorem $4.1 u_{h}-\omega(|h|)$ is a subminimum of $I$ on $\Omega_{h}$ and thus on $\Omega \cap \Omega_{h}$ whereas $u$ is still the maximum of the minimizers of $I$ on $\Omega \cap \Omega_{h}$. The Comparison Principle (Thm. 5.1) implies that $u_{h}-\omega(|h|) \leq u$ a.e. on $\Omega \cap \Omega_{h}$. Now let $x, y$ be two Lebesgue points of $u$ and let $r>0$ be such that $B_{r}(x)$ and $B_{r}(y)$ are contained in $\Omega$. Let $h=y-x$; since $u(z+h) \leq u(z)+\omega(|h|)$ for a.e. $z \in B_{r}(x)$, it turns out by integration on balls of radius $r$ and then passing to the limit as $r$ tends to 0 that $u(y)-u(x) \leq \omega(|y-x|)$; proving the claim. The case where $u$ is the minimum of the minimizers follows similarly. 
Remark 5.1. In $\left(\mathrm{H}_{2}\right)$ the regularity assumption on the boundary of $\Omega \cap(h+\Omega)$ is satisfied for instance if $\Omega$ is convex. In $\left(\mathrm{H}_{3}\right)$ it is more natural to choose $\omega$ as a common modulus of continuity of $\ell_{1}, \ell_{2}$.

Under a further mild assumption on $f$ it turns out that the conclusion of Theorem 5.2 holds for every minimum of $I$. We recall that a face of the epigraph of the convex function $f$ is the intersection of the epigraph with any of its supporting hyperplanes.

Corollary 5.1. Under the conditions of Theorem 5.2, assume moreover that the diameters of the projections onto $\mathbb{R}^{n}$ of the faces of the epigraph of $f$ are uniformly bounded by a constant $K$ and that $f$ is superlinear. Then any minimizer of $I$ is $\widetilde{\omega}$-continuous, where $\widetilde{\omega}(t)=\max \{K|t|, \omega(t)\}$.

Proof. Since $f$ is superlinear the maximum and the minimum of the minimizers of $I$ in $\phi+W_{0}^{1,1}(\Omega)$ exist: the proof of the claim given in [11], Proposition 4.1, actually holds true for any Lagrangian satisfying Tonelli's assumptions for the existence of a minimizer. Let $u$ be a minimizer and $U$ be the maximum of the minimizers of $I$ in $\phi+W_{0}^{1,1}(\Omega)$. From Theorem $5.2|U(y)-U(x)| \leq \omega(|y-x|)$ for every Lebesgue points $x, y$ of $U$. Moreover [10], Lemma 4.9, shows that $(\nabla u, f(\nabla u))$ and $(\nabla U, f(\nabla U))$ belong a.e. to the same face of the epigraph of $f$ : our assumption then implies that there is $K>0$ such that $|\nabla U-\nabla u| \leq K$ a.e. so that $u-U$ is Lipschitz of rank $K$. Thus for every Lebesgue points $x, y$ of $U$ we have

$$
\begin{aligned}
|u(y)-u(x)| & \leq|(u-U)(y)-(u-U)(x)|+|U(y)-U(x)| \\
& \leq K|y-x|+\omega(|y-x|) \leq \widetilde{\omega}(|y-x|),
\end{aligned}
$$

proving the claim.

As an application of the main result of this paper we obtain the following Hölder estimate.

Proposition 5.1. Let $\alpha \in] 0,1]$ and $\Omega$ be convex; let $\phi$ be Lipschitz in $\Omega$. Let $u$ be the minimum or the maximum of the minimizers of $I$ in $\phi+W_{0}^{1,1}(\Omega)$, or any minimizer if $f$ satisfies the assumptions of Corollary 5.1. Assume moreover that

$$
\forall \gamma \in \partial \Omega \quad|u(x)-\phi(\gamma)| \leq C|x-\gamma|^{\alpha} \quad \text { a.e. } x
$$

for some $C>0$. Then $u$ is Hölder continuous of order $\alpha$.

Proof. First notice that $\phi$ is Hölder continuous of order $\alpha$ : let $C^{\prime}$ be its Hölder constant. Set $\omega(t)=C^{\prime \prime}|t|^{\alpha}$ where $C^{\prime \prime}=\max \left\{C, C^{\prime}\right\}$. Since $\Omega$ is convex the Assumption $\left(\mathrm{H}_{2}\right)$ of Theorem 5.2 is satisfied, proving the claim.

Remark 5.2. Proposition 5.1 provides an alternative and more concise proof of one of the key points of the proof of the Hölder regularity result stated in [12], Theorem 4.5. The result was obtained there via a translation method inspired by [2].

\section{REFERENCES}

[1] H. Brezis, Analyse fonctionnelle : théorie et applications. Collection Mathématiques Appliquées pour la Maîtrise [Collection of Applied Mathematics for the Master's Degree], Masson, Paris (1983).

[2] H. Brezis and M. Sibony, Équivalence de deux inéquations variationnelles et applications. Arch. Rational Mech. Anal. 41 (1971) 254-265.

[3] A. Cellina, On the bounded slope condition and the validity of the Euler Lagrange equation. SIAM J. Control Optim. 40 (2002) 1270-1279.

[4] A. Cellina, Comparison results and estimates on the gradient without strict convexity. SIAM J. Control Optim. 46 (2007) 738-749.

[5] F. Clarke, Continuity of solutions to a basic problem in the calculus of variations. Ann. Sc. Norm. Super. Pisa Cl. Sci. 4 (2005) 511-530.

[6] L.C. Evans and R.F. Gariepy, Measure theory and fine properties of functions. Studies in Advanced Mathematics, CRC Press, Boca Raton (1992). 
[7] M. Giaquinta and L. Martinazzi, An introduction to the regularity theory for elliptic systems, harmonic maps and minimal graphs, Scuola Normale Superiore di Pisa (Nuova Serie) [Lecture Notes. Scuola Normale Superiore di Pisa (New Series)] 2. Edizioni della Normale, Pisa (2005).

[8] P. Hartman, On the bounded slope condition. Pacific J. Math. 18 (1966) 495-511.

[9] P. Hartman and G. Stampacchia, On some non-linear elliptic differential-functional equations. Acta Math. 115 (1966) $271-310$.

[10] C. Mariconda and G. Treu, Lipschitz regularity for minima without strict convexity of the Lagrangian. J. Differ. Equ. 243 (2007) 388-413.

[11] C. Mariconda and G. Treu, Local Lipschitz regularity of minima for a scalar problem of the calculus of variations. Commun. Contemp. Math. 10 (2008) 1129-1149.

[12] C. Mariconda and G. Treu, Hölder regularity for a classical problem of the calculus of variations. Adv. Calc. Var. 2 (2009) 311-320.

[13] M. Miranda, Un teorema di esistenza e unicità per il problema dell'area minima in $n$ variabili. Ann. Scuola Norm. Sup. Pisa 19 (1965) 233-249.

[14] G. Treu and M. Vornicescu, On the equivalence of two variational problems. Calc. Var. Partial Differential Equations 11 (2000) 307-319. 